\title{
The effect of a zero-diversion policy on emergency department performance measures
}

\author{
Eman Spaulding ${ }^{1}$, Laurie Byrne ${ }^{1}$, Eric Armbrecht ${ }^{2}$, Collin J ackson ${ }^{1}$, Preeti Dalawari ${ }^{1}$ \\ 1. Division of Emergency Medicine, Saint Louis University School of Medicine, Saint Louis, Missouri, United States. \\ 2. Ceneter for Outcomes Research, Saint Louis University, Saint Louis, Missouri, Unites States.
}

Correspondence: Preeti Dalawari. Address: School of Management, Division of Emergency Medicine, 3635 Vista at Grand Avenue, First Floor Desloge Towers, Saint Louis, Missouri 63110. E-mail: pdalawar@slu.edu

Received: May 31, 2013

DOI : $10.5430 /$ jha.v2n4p144

Online Published: August 21, 2013

Accepted: August 9, 2013

Online

\section{Abstract}

This study examines how emergency department (ED) performance measures at an academic tertiary care center in the Midwest were affected by a regionally-adopted zero diversion policy.

Two six-month periods before and after the policy was enacted were selected to measure differences in key performance measures, including left without treatment (LWOT), left without being seen (LWBS), left against medical advice (AMA), mortality, length of stay and hospital admission rate.

Total ED census during the two periods was similar. While the zero diversion policy was in effect, LWOT and LWBS rates were $19.4 \%$ and $18.2 \%$ lower, respectively, than the prior period, $p<.002$; discharged patients had faster treatment times $(228+8.0$ minutes vs. $242+9.0$ minutes $), p=.015$. No differences were observed in AMA or mortality rates. This study revealed no worsening of ED performance measures after adoption of a zero diversion policy.

\section{Key words}

Ambulance diversion, Hospital performance measures

\section{I ntroduction}

Ambulance diversion is a controversial and highly discussed issue within the practice of emergency medicine. When an emergency department (ED) goes on diversion, emergency medical service vehicles transport away to other EDs in the city. Theoretically, these "breaks" in patient arrival during diversion would allow the department to recover to a more optimal state of patient care/preparedness for a major disaster. Initially, ambulance diversion was intended to help the problem of ED overcrowding ${ }^{[1]}$. Contrary to the original intention, diversion has become commonplace and is used frequently across the country at many EDs. Approximately 45\% of United States (US) EDs reported diverting ambulances at some point within the previous year. Of those EDs, $3 \%$ of their total operating time was spent on diversion ${ }^{\text {[2] }}$. While there are many anecdotal reports, little data exists regarding the association between diversion and patient mortality, safety, or care ${ }^{[3-5]}$. One recent study showed a relationship between prolonged ambulance diversion and increased mortality in patients with myocardial infarction ${ }^{[6]}$. 
ED overcrowding is a serious problem reported by more than $90 \%$ of surveyed ED directors related to issues such as high patient acuity, hospital bed shortage, excessive wait times, higher ED and patient volumes, laboratory and radiology delays, and staffing shortages ${ }^{[7]}$. Even resident education has been a concern ${ }^{[8-10]}$. A report by the Institute of Medicine in 2007 revealed increases in ED utilization with decreases in capacity ${ }^{[11]}$, and one study showed that a diversion plan relieved the strain of overcrowding on staff and resources by rare and judicious use of ambulance diversion for short periods of time ${ }^{[12]}$. This concept was further explored in San Diego, California in an effort to decrease ambulance diversion, and it was found that decreasing diversion can be achieved with efforts of "collaboration and implementation of best practices,” including improving patient triage and efforts to get patients admitted more quickly ${ }^{[13]}$.

\section{Goal of this investigation}

The purpose of this study is to evaluate the effect of a zero diversion policy on ED performance measures and patient outcomes at a major trauma center in an urban Midwest area. We hypothesized that there would be no difference in ED performance, as measured by standard operating measures, under a zero-diversion policy in comparison to a policy in which the hospital had the option to activate a diversion status.

\section{Methods}

\subsection{Research approach}

This study employs an observational design in which hospital-level measures are compared before and after an intervention (aka, policy change). The hospital's pre-intervention phase serves as the comparison. The research question and setting does not permit controlling for confounding variables both internal and external to the hospital under study.

\subsection{Study design}

To assess the potential effect of a no-diversion policy on ED performance and patient outcomes, we constructed a retrospective study using administrative data to permit comparisons between two policy periods - hospital-option vs. zero diversion. The zero diversion was initiated on October 1, 2009. The six months immediately preceding the policy changes (April 1, 2009 - September 30, 2009) were selected to represent the hospital-option period, a period of time in which the hospital could opt to activate an "on diversion" status which would prevent ambulances from delivering additional patients to the ED. Prior to October 1, 2009, the Saint Louis University Hospital (SLUH) ED could activate diversion once the ED reached $120 \%$ saturation, which is determined through a calculation based on number of occupied/total beds, number of patients in the waiting room, number of patients en route, number of holding admitted patients, and staffing. To limit the effect of seasonal variation in acuity and volume, the zero diversion policy-period is represented by the same calendar months following the policy change (April 2010-September 2010). This study was approved by our Institutional Review Board of the investigators.

\subsection{Setting}

The data was collected at an inner city Level 1 academic university setting with an approximate ED annual volume of 36,000 patients and a 30\% admission rate. According to the Emergency Medicine Benchmarking Alliance, the hospital is a high acuity ED (admission level greater than 20\%, presence of a transplant service, and Level 1 trauma designation) ${ }^{[14]}$.

\subsection{Data collection}

Data was obtained from the hospital's administrative database which provides standard monthly reporting for each indicator. ES and CJ reviewed these records and entered the data points into a Microsoft excel spreadsheet. LB and EA reviewed all data points for accuracy. 


\subsection{Outcome measures}

The main outcomes were five indicators of ED performance and two indicators of patient outcomes. The ED performance measures included were: (a) left without treatment (LWOT), defined as a patient who left the ED before being treated, (b) left without being seen (LWBS), defined as a patient who signed into the ED but was never triaged, (c) left against medical advice (AMA), defined as a patient who left the ED despite being advised otherwise by the attending ED physician, (d) length of stay for discharged patients (LOSD), and (e) length of stay for admitted patients (LOSA), which are the times in minutes that patients were in the ED before either being discharged home or admitted to the hospital. The two patient outcome indicators were hospital admission rate (HAR) and mortality. All indicators were selected based on their routine use in reporting at the study hospital and common use among ED and hospital administrators to assess ED performance.

\subsection{Data analysis}

A two-sided z-ratio was used to test the difference between hospital-option and zero diversion policy periods for LWOT, LWBS, AMA, deaths, and admission rate. Z-ratios were used to test the differences in proportion. Rates were expressed as per 1,000 ED patients visits. All people who presented to the ED are included in the ED patient count denominator. Differences in length of stay (LOSD and LOSA) were assessed with a $t$-test for independent samples. An alpha of 0.05 was selected as a threshold for significance for all statistical tests.

Data analysis was performed with PASW Statistics 18 (SPSS: An IBM Company) and VassarStats, a web-based statistical calculation tool (http://faculty.vassar.edu/lowry/VassarStats.html).

\section{Results}

The total number of patient visits to the ED during the hospital-option and zero diversion periods (of six months each) were approximately the same at 18,108 and 18,698, respectively. During the hospital-option period, diversion was activated for an average of 7.0 hours per month (range 1.3 hours to 16.8 hours per month), or about $1 \%$ of all hours during which the ED was operational.

\subsection{Emergency department performance}

For three of the five indicators of ED performance, statistically significant differences between hospital-option and zero diversion periods were observed, and all measures were indicative of more favorable performance during zero diversion.

Table. ED performance measures during hospital option vs. zero diversion

\begin{tabular}{llll}
\hline \multirow{2}{*}{ ED Performance Measures } & Hospital Option & Zero Diversion & $\boldsymbol{p}$ value \\
\hline LWOT* & $\mathbf{N = 1 8 , 1 0 8}$ & $\mathbf{N = 1 8 , 6 9 8}$ & $<.001$ \\
LWBS* & 70.8 & 57.1 & .002 \\
AMA* & 28.7 & 23.5 & .183 \\
Death* & 13.3 & 11.7 & .653 \\
Admitted Patients* & 3.5 & 3.2 & .009 \\
& 280.59 & 292.87 & .496 \\
Admitted Patients & \multicolumn{2}{c}{ Length of Stay, minutes (mean +/- SD) } \\
Discharged Patients & $334+/$ - 11 & $329+/-12$ & .015 \\
\hline
\end{tabular}

*rate per 1,000 patients

As seen in Table, LWOT was 70.8 per 1,000 census during hospital-option and 19.4\% less (at 57.1 per 1,000) during zero diversion, $p<.001$. Similarly, LWBS rates were 18.2\% during the zero diversion period (23.5 per 1,000 census) in comparison to the hospital-option (28.7 per 1,000 census), $p=.002$. Patients discharged from the ED had shorter lengths of 
stay (LOSD) during the zero diversion $(228+8.0$ minutes) $v$ s. hospital-option $(242+9.0$ minutes), $p=.015$. There were no differences between the policy periods in observed AMA rates $(p=.183)$ or ED length of stay among patients who were admitted to the hospital (LOSA), $p=.496$ (see Table).

\subsection{Patient outcomes}

HAR was $4.4 \%$ higher during zero diversion ( $p=.009$ ); 293 per 1,000 vs. 281 per 1,000. There were no differences in mortality rates between the two study periods $(p=.653)$.

\section{Discussion}

Factors contributing to ED crowding are broadly categorized as input, throughput, and output; the most influential factor is output (e.g. the practice of holding or "boarding" admitted patients in the ED for several hours to days because of decreased inpatient capacity). Diversion, on the other hand, is an input factor, and has not been proven to relieve ED congestion and has been theorized to be less important of a factor ${ }^{[15]}$. Ultimately, evidence for or against diversion is limited at best, and we believed there would be no statistical difference in emergency department performance measures and outcomes between diversion and no-diversion. Among the Houston, Texas EMS, the percentage of deaths among all trauma patients, transfers, and non-transfers admitted on days with substantial diversion activation was consistently higher, but the difference was not statistically significant ${ }^{[16]}$. In our area ambulances transported unstable patients to the closest ED despite diversion status during the hospital-option period. Therefore, increases in mortality attributable to diversion may be limited since the hospital-option period did not consistently prevent unstable patients from accessing the closest facility in our area. Despite the higher ED volume, there was no increase in AMA, LWOT, LWBS, or mortality.

The most promising data may be from a study from Western Massachusetts, which was a multicenter trial that analyzed two 3-month periods of diversion and no diversion. They showed that a diversion ban was not associated with significant changes in "throughput measures" in EDs; these measures being total volume, number of admissions, number of elopements, and length of stay for all patients, length of stay for admitted patients, and length of stay for discharged patients ${ }^{[17]}$.

In our study, there was an improvement in average length of stay for discharged patients from the ED, LWOT and LWBS during zero diversion $v$ s. hospital-option. While LWOT and LWBS are indicators of substantial overcrowding, we did not control directly for overcrowding, which is a dynamic function of ED volume and staffing capacity. These two performance measures are clinically relevant for a systems-based practice like our ED because staff and physician support for a zero-diversion policy depends on clear indicators that the policy did not result in overcrowding. This data suggests that the zero diversion policy is not negatively associated with these measures; however, the improvement noted is difficult to explain (and likely multifactorial), considering that there were no major inpatient policy changes (such as increase in ICU bed availability, or system to improve throughput by decreasing boarding patients), staffing changes in nurses, midlevel providers, attending physician coverage in the ED, or operational ED changes to improve patient flow or mortality.

The one key difference between the two time frames was that an emergency medicine residency program was started on July 1, 2009. A study by McGarry et al., in a similar size urban hospital, showed that the presence of emergency medicine residents decreased LWBS (but not by a significant margin), while admission rates and LOS were relatively similar ${ }^{[18]}$. While interns are not in the ED for a substantial amount of time their first year, the fact that the zero diversion policy period was a transition point in that there were currently two years of residents as opposed to one year and thus more ED residents, may have accounted for these improvement in measures.

Literature on LWBS suggests that these patients are generally lower acuity; thus it is reasonable that we saw improvement in all three indicators (i.e.- if LOS for discharged patients improved, then LWBS and LWOT may improve as well) ${ }^{[19]}$. 
Also, the National Center for Health Statistics note that ambulance diversion increases wait times for urgent, semi-urgent, and non-urgent conditions, with no effect on emergent or immediate condition wait times ${ }^{[20]}$. As a proxy, increased wait times lead to increased LOS times; so it is possible that these wait times and thus LOS of discharged patients were affected in the no diversion policy period.

Although counter intuitive, other studies are showing improvements in measures as well. Data from nine hospitals in Massachusetts observed a decrease in length of stay for admitted patients and decrease in ambulance turnaround time after the implementation of their statewide no diversion policy. In fact, hospitals with high diversion rates actually had a decreased LOS for discharged patients, although not statistically significant. Like our study, these studies suggest that a diversion ban does not necessarily lead to ED overcrowding ${ }^{[15,17]}$. However, Burke et al. was able to point to concrete interventions that were made by hospitals in changes to practice such as inpatient bed rounds twice a day to decompress the ED, reduction in target hospital occupancy level, and prioritization of inpatient bed assignments, to name a few.

Another factor to consider is that ED patient flow is not only a systems issue. In fact, one source says that it is $50 \%$ systems issue, and $50 \%$ physician issue ${ }^{[21]}$. Because policy changes in hospitals rarely happen discreetly, the fact that the physicians and nurses knew this policy change would be coming may have affected their work. It is possible, knowing there would be no reprieve by diversion, physicians and staff modified their workups, delegated tasks more judiciously, streamlined management decisions such as ordering tests all at once, and perhaps admitted patients more quickly (iewithout all laboratory work back), etc.

\subsection{Limitations}

There are some limitations to this study. First, data was collected for only one institution and thus does not completely reflect the experiences at the local city-wide level with multiple institutions involved in this policy. It is also difficult to assess the regional and then nationwide effects of a zero diversion policy. Because this data cannot take into account separate ED volumes across the nation, it cannot speak to how a zero-diversion policy may apply to a larger metropolitan or community areas. Second, while we have data as it pertains to diversion vs. zero-diversion, the ED is not always overcrowded, thus this data cannot be applied to measurable outcomes in general. Since we have not directly controlled for overcrowding, it would be worth knowing how clinical outcomes are affected during zero-diversion when the ED is overcrowded versus when the ED is not overcrowded. Third, while there was a significant decrease in the average length of stay for discharged patients, and a decrease in the rates for patients who LWOT and LWBS, we hypothesized that there would be no difference in these outcome measures during the periods studied, and are currently unable to definitively account for this improvement with this administrative data. While we have some theories to account for the trend, ED flow, efficiency, and overcrowding are multifactorial issues, for which measurements were not made as part of the scope of this study. These factors could account for some or much of the observed changes. Furthermore, although mortality was not reported as risk adjusted, our acuity levels via our triage scoring system were similar between both time periods. Finally, the low baseline diversion rate may be a limitation in applying these findings to other settings where diversion practices are more common.

\subsection{Future direction}

First, while statistically significant findings were noted for ED outcomes and average length of stay for patients discharged from the ED, analysis of outcomes annually would be useful to note if there is sustained improvement of our clinical practice based management and the potential impact of a no diversion policy. The effect of physician triage on ED diversion has been a topic of discussion as well. Data published in 2009 on the effect of physician triage on ED length of stay at Vanderbilt University Medical Center showed an average decrease in days on diversion per month ${ }^{\text {[22] }}$. Second, there were no specific interventions such as changes in ED staffing or inpatient boarding policies that explain why these indices changed significantly while on zero-diversion. It would be interesting to delve deeper into the factors that affect physician efficiency in the ED that may have accounted for some of the observed effect. 
Finally, this study identified LWOT, LWBS, AMA, and deaths as outcomes pre- and post-diversion, as well as admissions and length of stay for patients in the ED. While these are standard quality assurance parameters, there are others worth considering. For example, leveled trauma volume, ED staff efficiency, and triage category could also be measured. Further issues to consider are the cost of diversion opposed to zero-diversion. A cost analysis was not done pre- and post-diversion to determine the financial implications of a zero-diversion policy, nor its affect on ED resources.

\section{Conclusions}

Adopting a zero diversion policy was not associated with increased rates of death, AMA or overall ED length of stay at this single mid-sized, high acuity ED. While our results revealed improvements in key performance measures, including rates of patients leaving without being seen or treated and decreased length of stay for discharged patients, these findings were unexpected and further research needs to be done to analyze what factors, if any, can account for the aforementioned results.

The results of this study may inform decision makers in developing new models of diversion within hospitals and open community wide dialogue about a zero diversion policy. It is our hope that this research can be used as a framework for other institutions to evaluate the effect of diversion on their ED and contribute to evidence that further explores the association between diversion and ED performance and patient outcomes.

\section{References}

[1] Pham, J. C., Patel, R., Millin, M. G., Kirsch, T. D., Chanmugam, A. The effects of ambulance diversion: a comprehensive review. Acad Emerg Med. 2006; 13(11): 1220-1227. http://dx.doi.org/10.1197/j.aem.2006.05.024

[2] Burt, C. W., McCaig, L. F., Valverde, R. H. Analysis of ambulance transports and diversions among US emergency departments. Ann Emerg Med. 2006; 47(4): 317-326. http://dx.doi.org/10.1016/j.annemergmed.2005.12.001

[3] Pines, J. M., Localio, A. R., Hollander, J. E., Baxt, W. G., Lee, H., Phillips, C., et al. The impact of emergency department crowding measures on time to antibiotics for patients with community-acquired pneumonia. Ann Emerg Med. 2007; 50(5): 510-516. http://dx.doi.org/10.1016/j.annemergmed.2007.07.021

[4] Schull, M. J., Morrison, L. J., Vermeulen, M., Redelmeier, D. A. Emergency department gridlock and out-of-hospital delays for cardiac patients. Acad Emerg Med. 2003; 10(7): 709-716. PMid: 12837644. http://dx.doi.org/10.1111/j.1553-2712.2003.tb00064.x

[5] Shenoi, R. P., Ma, L., Jones, J., Frost, M., Seo, M., Begley, C. E. Ambulance Diversion as a Proxy for Emergency Department Crowding: The Effect on Pediatric Mortality in a Metropolitan Area. Academic Emergency Medicine. 2009; 16(2): 116-123. http://dx.doi.org/10.1111/j.1553-2712.2008.00317.x

[6] Shen, Y. C., Hsia, R. Y. Association Between Ambulance Diversion and Survival Among Patients With Acute Myocardial Infarction. Jama-Journal of the American Medical Association. 2011; 305(23): 2440-2447.

http://dx.doi.org/10.1001/jama.2011.811

[7] Pines JM, Hollander JE. Emergency Department Crowding is Associated With Poor Care for Patient With Severe Pain. Ann Emerg Med. 2008; 51(1):1-5. PMID: 17913299. http://dx.doi.org/10.1016/j.annemergmed.2007.07.008

[8] Derlet, R., Richards, J., Kravitz, R. Frequent overcrowding in U.S. emergency departments. Acad Emerg Med. 2001; 8(2): 151-155. PMid: 11157291. http://dx.doi.org/10.1111/j.1553-2712.2001.tb01280.x

[9] Mahler, S. A., McCartney, J. R., Swoboda, T. K., Yorek, L., Arnold, T. C. The impact of emergency department overcrowding on resident education. J Emerg Med. 2012; 42(1): 69-73. http://dx.doi.org/10.1016/j.jemermed.2011.03.022

[10] Harris, A., Sharma, A. Access block and overcrowding in emergency departments: an empirical analysis. Emerg Med J. 2010; 27(7): 508-511. http://dx.doi.org/10.1136/emj.2009.072546

[11] Committee on the Future of Emergency Care in the United States Health System. Institute of Medicine. Hospital-Based Emergency Care: At the Breaking Point. Report from Washington, DC: Institute of Medicine. The National Academies Press, Washington, D.C. 2007. Retreived at: http://www.nap.edu/catalog.php?record_id=11621\#toc 
[12] Anderson E, Riddle K, Bear R. Results of implantation of a contemporary model for ambulance diversions in an integrated healthcare delivery system. Healthcare Management Forum. 1999; 12(4): 49-50. PMID: 10788072. http://dx.doi.org/10.1016/S0840-4704(10)60168-8

[13] Castillo, E. M., Vilke, G. M., Williams, M., Turner, P., Boyle, J., Chan, T. C. Collaborative to decrease ambulance diversion: the California Emergency Department Diversion Project. J Emerg Med. 2011; 40(3): 300-307. http://dx.doi.org/10.1016/j.jemermed.2010.02.02

[14] Emergency Department Performance Measures and Benchmarking Summit: The Consensus Statement. Retrieved on August 5, 2013, from http://urgentmatters.org/media/file/EDBA.pdf

[15] Burke, L. G., Joyce, N., Baker, W. E., Biddinger, P. D., Dyer, K. S., Friedman, F. D., et al. The effect of an ambulance diversion ban on emergency department length of stay and ambulance turnaround time. Ann Emerg Med. 2013; 61(3): 303-311 e301. http://dx.doi.org/10.1016/j.annemergmed.2012.09.009

[16] Begley CE, Chang Y, Wood RC, Weltge A. School of Public Health, University of Texas Health Science Center, Houston, Texas. Emergency department diversion and trauma mortality: evidence from Houston, Texas. J Trauma. 2004; 57(6):1260-1265. PMID: 15625459. http://dx.doi.org/10.1097/01.TA.0000135163.60257.A6

[17] Rathlev, N. K., Blank, F., Osborne, B., Kellogg, A., Li, H. P., Blanchet, J., et al. No Diversion in Western Massachusetts. Journal of Emergency Medicine. 2013; 44(2): 313-320. http://dx.doi.org/10.1016/j.jemermed.2012.06.017

[18] McGarry, J., Krall, S. P., McLaughlin, T. Impact of resident physicians on emergency department throughput. West J Emerg Med. 2010; 11(4): 333-335. PMid: 21079704.

[19] Kennedy, M., MacBean, C. E., Brand, C., Sundararajan, V., Mc, D. T. D. Review article: Leaving the emergency department without being seen. Emerg Med Australas. 2008; 20(4): 306-313. http://dx.doi.org/10.1111/j.1742-6723.2008.01103.x

[20] Hing, E., Bhuiya, F. Wait time for treatment in hospital emergency departments: 2009. NCHS Data Brief. 2012 ; (102): 1-8.

[21] American College of Physicians: Clinical \& Practice Management. (2004). Efficiency in the Emergency Department. Retrieved August 5, 2013, from http://www.acep.org/content.aspx?id=29876

[22] Han, J. H., France, D. J., Levin, S. R., Jones, I. D., Storrow, A. B., Aronsky, D. The effect of physician triage on emergency department length of stay. J Emerg Med. 2010; 39(2): 227-233. http://dx.doi.org/10.1016/j.jemermed.2008.10.006 